\title{
Developing a Baseline for Upper-Body Motor Skill Assessment using a Robotic Kinematic Model*
}

\author{
Sergio García-Vergara, Student Member, IEEE, Miguel M. Serrano, Student Member, IEEE, Yu-Ping \\ Chen, and Ayanna M. Howard, Senior Member, IEEE
}

\begin{abstract}
In the rehabilitation field, determining the effectiveness of an intervention protocol begins by comparing the individual's movement characteristics against a baseline. In most settings, this baseline is determined through clinical studies involving a range of patients belonging to the same demographic group. Unfortunately, this leads to a process that is difficult to repeat for all patient demographics, or all movement characteristics, given the demands on clinicians' and patients' time for performing such clinical baseline measurement studies. To address this issue, we discuss a method that allows clinicians to objectively assess an individual's movements and compare the resulting outcome kinematic metrics to a kinematic baseline. Instead of collecting human patient data, we propose a robotic kinematic model that generates a baseline for different kinematic parameters in real-time as a function of the state of a given task. We evaluate our methodology on elbow and shoulder range of motion (ROM) angles obtained from eleven typically developing children. We compare the user's ROM angles to those generated by the proposed model, and discuss the potential of the model to be used in various intervention protocols.
\end{abstract}

Keywords - robotic rehabilitation, physical therapy, upperbody assessment, kinematic model

\section{INTRODUCTION}

In general, individuals with a motor skill disorder are required to engage in some form of physical therapy. Some common disorders include, but are not limited to: cerebral palsy, Parkinson's disease, and motor impairment due to stroke. In addition to the exercises performed at the clinical center, physical therapy protocols require individuals to perform a certain set of recommended exercises in their homes in order to avoid further development of any existing symptoms and to shorten the time of recovery [1]. In both cases, whether for in-home or clinic-based therapy protocols, clinicians must be able to assess progress in the patient's motor skill in order to determine the effectiveness of the protocol and modify if necessary. To perform this assessment, there are a number of different processes that can be employed.

Previous research has shown the positive use of robotaided rehabilitation systems in objectively assessing human movement in therapy scenarios ranging from stroke rehabilitation [2] [3] [4] to motor development in children [5]. Krebs et al. [2] presented an approach to analyze kinematic data collected using a prototype robot-aided rehabilitation facility. Not only did they show that robot-aided therapy

* Research partially supported by the National Science Foundation under Grant No. IIS-1208287, and by the NSF Graduate Research Fellowship under Grant No. DGE-1148903.

S. García-Vergara, M. M. Serrano, and A. M. Howard are with the School of Electrical and Computer Engineering, Georgia Institute of Technology, presents no adverse effects on patients, but also that the combination of robotics and automation with concepts of neuroscience has the potential to allow for the development of better kinematic assessment tools. Volpe et al. [3] showed that individuals who acquired additional sensorimotor training delivered by a robotic device demonstrate improved functional outcome and enhanced motor performance. Clinical trials developed by Burgar et al. [4] showed that the motor recovery improvements achieved by individuals who participate in robot-assisted therapy sessions exceed the improvements achieved by those who participate in traditional therapy sessions. In [5], Galloway et al. studied the possibility of using mobile robots as part of the rehabilitation of young infants with special needs. Finally, Colombo et al. [6] developed a robotic device to fulfill the need to assess the performance of an individual through repeatable and quantifiable metrics as an effective means for rehabilitation.

Such methodologies, as discussed, have proven to be successful in aiding rehabilitation therapists in various rehabilitation settings. However, due to hardware complexity and their contact-based approach, these systems are largely restricted to be used in the clinical setting and are not practical options for in-home assessment with untrained users. In order to expand such assessment methods to in-home settings, some researchers have looked at non-contact based methods for kinematic assessment. Howard et al. [7] compared the benefits of non-contact versus contact sensing methodologies for inhome rehabilitation. They concluded that, primarily due to cost and the complexity of the system, it would be challenging to integrate the required hardware in most real-world human settings for the contact-based approach. Brooks et al. [8] developed an objective and quantifiable methodology for assessing upper-body movements using computer vision techniques. However, the method required the user's sagittal plane to be perpendicular to the camera's focal vector in order for the algorithm to capture the needed images, and for the user's elbow joint to be locked throughout any movement made. Unfortunately, these assumptions limit the types of interactions required by a user to interact successfully with a given system - for example a virtual gaming platform.

Another way of quantifiably assessing an individual's movement data is to compare the resulting outcome metrics to a baseline. Unlike the aforementioned methods, this is a welldocumented evidence-based practice found widely in the practicing clinical literature. Butler et al. [9] collected data

Atlanta, GA 30332 USA (emails: sergio.garcia@gatech.edu, ayanna.howard@ece.gatech.edu,mserrano6@gatech.edu).

Y. P. Chen is with the College of Health \& Human Science, Division of Physical Therapy, Georgia State University, Atlanta, GA, 30302 USA (email: ypchen@gsa.edu). 
from 25 typically developing children, and generated a baseline that describes healthy upper-body movements. Troke et al. [10] developed a normative database on indices of ranges of motion in the lumbar spine, allowing for the potential of it being used as a baseline in rehabilitation protocols. Another commonly used kinematic database, developed at the Children's Hospital, San Diego [11], contains temporal-spatial and joint angle data for 409 gait cycles for children. Although results show that generating databases and comparing them to an individual's outcome metrics is an effective way to evaluate the resulting kinematic performance, generating the baseline based on human data can be time consuming and can have large variations due to the fact that not all individuals have similar movements. Moreover, baselines are generally relevant only to a specific scenario. If the tasks and/or kinematic metrics of interest are modified, a new baseline would need to be created.

As such, to combine the clinical-based practice of using baseline data for assessment and the advantages of non-contact based sensing methods, we focus on developing a non-contact based methodology that is capable of quantifiably comparing an individual's kinematic metrics to a baseline generated in real-time, allowing for in-home kinematic assessment. Section II describes in detail the proposed kinematic model used to develop a baseline. Section III describes the definitions and equations used to calculate an individual's kinematic metrics, and discusses the process for comparing these measures to the generated baseline. Section IV compares the outcome of the model with real-world kinematic data derived from human participants. Finally, Section $\mathrm{V}$ provides observations and concluding remarks.

\section{Methodology}

In this paper we discuss a kinematic model that is capable of generating a baseline for different motor skill parameters. Currently, to the best of our knowledge, there is not a system that evaluates an individual's movements in real-time by quantitatively comparing the resulting motor skill parameters to those generated by a kinematic model, eliminating the need to create a baseline with human data. Such a system would also enable evaluation for in-home rehabilitation, which would assist in evaluating intervention protocols outside of the clinical setting.

For simplicity, we focus on upper-body movement parameters, of which the most dominant form is reaching movements. The ability to reach is critical for most, if not all, activities of daily living such as feeding, grooming, and dressing [12]. Moreover, failure to substantially recover upper-extremity function can lead to depression [13]. As such, reaching movements, correlated to reaching exercises, are of interest in various rehabilitation scenarios. These exercises require an individual to move from a defined initial position to a selected target position (Figure 1). To create a baseline model for this movement, and various other therapy-based movements, we must first define a model that is equivalent to human arm movement dynamics. We create a baseline model for motor skill assessment by constructing a kinematic chain of links that correlates to the dynamics of the human arm.

To model the human arm as a kinematic chain, certain assumptions about its motion and simplifications have to be made. The shoulder and elbow joints are represented as the joints of a two link kinematic chain (Figure 2), where $\theta_{i}$ is the angle of the ith joint, and $\mathrm{L}_{\mathrm{j}}$ is the length of the jth link. The model includes one spherical joint (shoulder) and one revolute joint (elbow). This means that the elbow has only one degree of freedom (DOF) while the shoulder has three. To reduce the complexity of the model, the wrist joint is modeled as part of the forearm/hand link. This simplification is valid because, in general, users don't move their wrists to reach a target during a reaching motion. Each transformation between joints is modeled using lie groups. Exploiting the lie group's structure, which accounts for both the link lengths and DOF, the user's wrist position, $g_{w}$, can be estimated using forward kinematics as shown in (1).

$$
\begin{aligned}
g_{w}= & {\left[\begin{array}{cc}
R_{z}\left(\theta_{1}\right) & {\left[\begin{array}{l}
0 \\
0 \\
0
\end{array}\right]} \\
1
\end{array}\right] *\left[\begin{array}{cc}
R_{y}\left(\theta_{2}\right) & {\left[\begin{array}{l}
0 \\
0 \\
0
\end{array}\right]} \\
0 & 1
\end{array}\right] *\left[\begin{array}{c}
R_{x}\left(\theta_{3}\right)\left[\begin{array}{l}
0 \\
0 \\
0
\end{array}\right] * \ldots \\
0
\end{array}\right] * } \\
& {\left[\begin{array}{cc}
R_{x}\left(\theta_{4}\right) & {\left[\begin{array}{c}
0 \\
0 \\
-L_{1}
\end{array}\right]} \\
1
\end{array}\right] *\left[\begin{array}{cc}
I_{3 x 3} & {\left[\begin{array}{c}
0 \\
0 \\
-L_{2}
\end{array}\right]} \\
0 & 1
\end{array}\right] }
\end{aligned}
$$

where $R_{x}, R_{y}, R_{z} \in S O(3)$ denote the rotation matrices along the $x, y, z$ axis respectively, $\theta_{i}$ is the angle of the ith joint, $L_{j}$ is the length of the jth link, and $\mathrm{I}_{3 \times 3}$ is a $3 \times 3$ identity matrix.

Using this kinematic model of the arm, we can solve for a trajectory from a start to a target point using the Penalized Manipulator Jacobian [14]. The Penalized Manipulator Jacobian is used to estimate the desired trajectory based on the initial and final wrist position. It minimizes the energy in (2).

$$
E=\frac{1}{2} *\|\zeta-J(d \theta)\|^{2}+\frac{1}{2} *\left\|d \theta^{T} * W * d \theta\right\|^{2}
$$

where $\mathrm{W}$ represents the penalization of the respective joint angles $(\theta), d \theta$ is the angle update, and $\zeta$ is the desired twist. This is a least squares problem and is thus a linear approximation of this complex problem. However, given a relatively small update it works very well for our problem.

With the model defined and the solution strategy in hand all that is left is to connect the initial pose to the desired wrist position given the Penalized Manipulator Jacobian. The subject's initial pose is estimated using the model and is set as the initial condition for the solver. For our system, the assumption was made that only the velocity components of the twist are required. We found this to be a fair assumption as the general motions typically involved direct translations of the subject's wrist, with little to no wrist rotation observed. The velocity components are represented by the first two components of $\zeta$ vector, and are equated as the normalized difference of the initial and final position of the end-effector. Solving for equation (2) at each iteration, $\mathrm{d} \theta$ is calculated and the joint angles are updated according to (3).

$$
\theta(k+1)=\theta(k)+\delta * d \theta(k)
$$

where $\theta(\mathrm{k})$ is the joint angle at the kth iteration, and $\delta$ is a constant from 0 to 1 . This process is continued until the hand position of the model has converged to the desired hand position (i.e. where the target is located). 

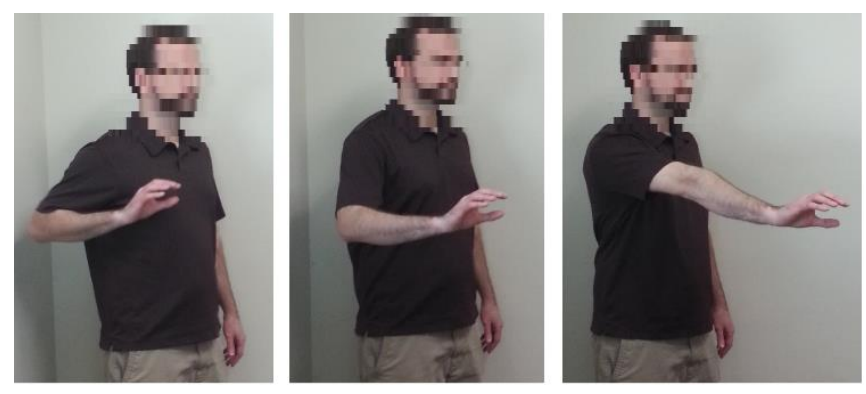

Figure 1. Sequence of snapshots showing an example of a typical 'reaching movement'.

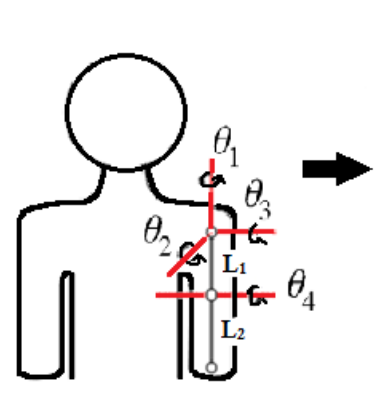

(a)

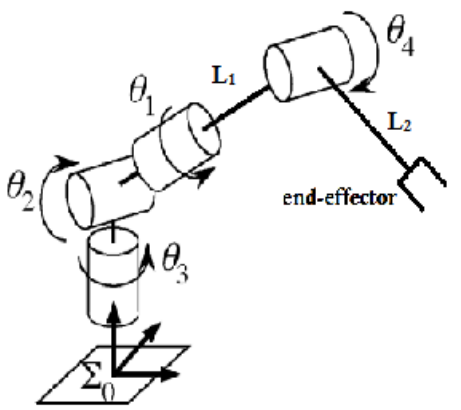

(b)
Figure 2. Mapping from (a) the human arm kinematics, to (b) the kinematics of a common 4 DOF robotic manipulator (adapted from [15]).

The model was designed such that the movements are not only feasible, but also efficient. The penalization values in $\mathrm{W}$ are fixed and empirically selected depending on whether the motion would prioritize a heavily-dependent elbow or shoulder motion. This prevents the model from creating trajectories that would require the subject to overextend themselves. The diagonal terms of the matrix $\mathrm{W}$ represent the corresponding weights for their respective joints. The magnitude of each weight is positive or equal to zero, with zero representing no penalization for that given joint motion. To prioritize the motion of a given joint, the magnitude of its weight must be an order different than that of the joints that are to be prioritized. For example, if the motion desired should prioritize an elbow motion, e.g. the target is located below the head of the user but near the body, then the elbow weight should be much lower than the weight of the shoulder. Similarly, for a target position located above the head, the shoulder should be prioritized and the weights defined accordingly.

\section{RANGE OF MOTION}

There are various kinematic parameters associated with upper-body motor skills, including movement smoothness [16], movement speed [17], and movement time [18]. In this paper, we focus on the user's range of motion (ROM). In the clinical setting, the ROM of a given joint is defined as the full (angular) movement potential of the joint, usually its range of flexion and extension [19]. In general, physical therapists are interested in evaluating an individual's joint flexibility. The ROM allowed for the joint of interest enables measurement of this flexibility parameter [20]. For upper-body movements,
ROM is associated with shoulder, elbow, and wrist movements, which can then be subdivided into abduction/adduction, and flexion/extension movements. For the shoulder joint, abduction/adduction is movement in the coronal plane whereas flexion/extension movements are in the sagittal plane (Figure 3). In this paper, we focus on evaluating the proposed baseline model on measurements of the elbow flexion and shoulder adduction ROM. We adhere to the angle references used in different rehabilitation scenarios similar to [21] as described by Figure 4. As per the definition in [18], for a given movement, the ROM of the corresponding joint is the difference between the final joint angle minus the initial joint angle. The elbow angle $\theta_{\mathrm{E}}$ is the dot product between the elbow-shoulder $(\overrightarrow{E S})$ and elbow-wrist $(\overrightarrow{E W})$ vectors as described by (4).

$$
\theta_{E}=\pi-\cos ^{-1}\left(\frac{\overrightarrow{E S} \cdot \overrightarrow{E W}}{|\overrightarrow{E S}| *|\overrightarrow{E W}|}\right)
$$

In general, therapists assess an individual's shoulder movement by restricting it to a single plane. Namely, the individual is asked to move the arm in the coronal or sagittal plane. In order to comply with regular physical therapy sessions, we project the relevant upper-body vectors onto the mentioned planes and then calculate the corresponding angles. The shoulder angle $\theta_{\mathrm{S}}$ in any plane is the angle between the user's shoulder-elbow vector $(\overrightarrow{S E})$ and the user's torso. For simplicity, we define a point $\mathrm{P}$ such that the shoulder-P vector $(\overrightarrow{S P})$ is always parallel to the user's torso (Figure 4). Regardless of the position of the user's arm, we project the shoulder-P and shoulder-elbow vectors onto the coronal and sagittal planes with (5). The final planar angles are calculates using the dot product as defined by (6).

$$
\begin{gathered}
\vec{P}=\vec{O}-\vec{n} *(\vec{O} \cdot \vec{n}) \\
\theta_{S}=\cos ^{-1}\left(\frac{\overrightarrow{S E} \cdot \overrightarrow{S P}}{|\overrightarrow{S E}| *|\overrightarrow{S P}|}\right)
\end{gathered}
$$

where $\vec{O}$ is the original vector, $\vec{P}$ is the projected vector, $\vec{n}$ is the normal vector of the plane where the original vector is being projected onto, and $\overrightarrow{S E}$ and $\overrightarrow{S P}$ are the shoulder-elbow and shoulder-P vectors respectively in their corresponding planes.

To determine a baseline metric, the distance between a start and target point and the user's arm link lengths are fed into the kinematic model, which uses them to generate the ROM baseline. Figure 5 shows an example of a user's trajectory compared to the trajectory generated by the kinematic model. Once the baseline is computed, the algorithm compares the user's ROM values to their respective baselines. The algorithm computes a difference percent error normalized by the corresponding joint's maximum capacity (7).

$$
R O M_{j, \text { error }}=\frac{\left|R O M_{j, \text { user }}-R O M_{j, \text { baseline }}\right|}{R O M_{j, \text { max }}} * 100 \%
$$

where $R O M_{j, \text { user }}$ is the user's ROM for joint $j, R O M_{j}$,baseline is the ROM computed by the kinematic model for joint $j$, and $\mathrm{ROM}_{\mathrm{j}, \mathrm{max}}$ is the maximum angle capacity for joint $\mathrm{j}$ (obtained by making a full joint movement). 


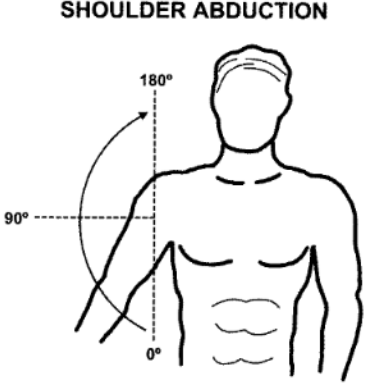

a b

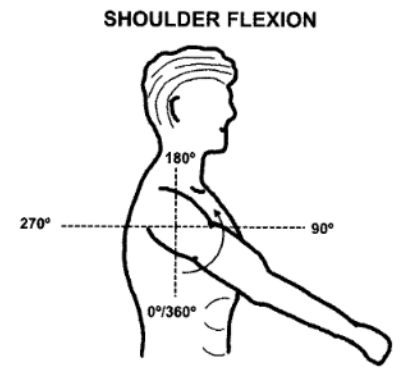

Figure 3. Examples of a) abduction movements in the coronal plane, and b) flexion movements in the sagittal plane. Adapted from [22].

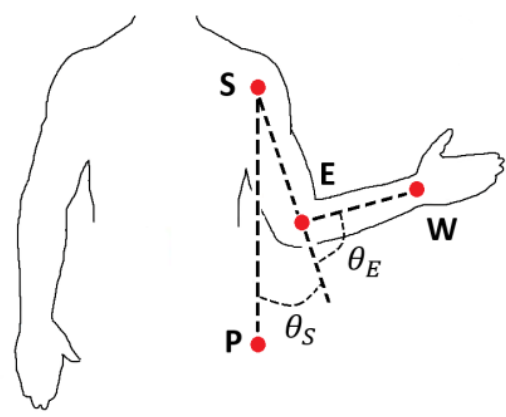

Figure 4. Diagram showing the used joints to calculate the elbow and shoulder range of motion (ROM). Figure shows an example of the ROM calculated for the coronal plane. (W - Wrist, E - Elbow, S - Shoulder, P Point in space below the shoulder joint, $\theta_{\mathrm{E}}-$ Elbow angle, and $\theta_{\mathrm{S}}-$ shoulder angle).

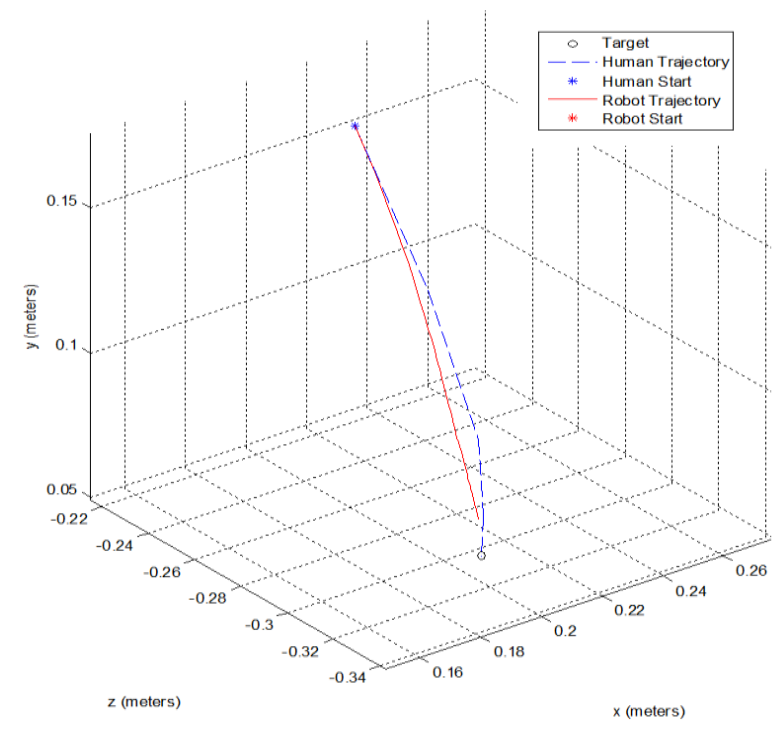

Figure 5. Comparison of the trajectory created by the user (dotted line), and the trajectory created by the robot model (continous line) moving from a shared initial position to the target.

\section{EXPERIMENTAL RESULTS}

The performance and accuracy of our methodology for creating a baseline model was evaluated with data collected from randomized trials with eleven typically developing children. Six females and five males, ranging in age between 6 and 11 years (mean age $=8.7$ years, standard deviation $=1.7$ years), were recruited for this study. The parents of the participants signed the IRB (Institutional Review Board) approved consent form allowing their children to engage in the testing sessions.

\section{A. Super Pop VR ${ }^{T M}$}

In order to enable collection of a non-biased data collection process for the randomized trails, we employed a platform called Super Pop VR ${ }^{T M}$ [23], [24], a motivating virtual reality game used to track upper-body movements using the Kinect camera from Microsoft. The objective of the Super Pop VR ${ }^{T M}$ game is to pop random bubbles that appear on screen; for the user, the execution of a reaching movement is necessary to successfully reach for and pop the 'bubble' (Figure 6). At various instances of time during game play, two (and only two) virtual bubbles are commanded to appear on the screen. The initial position of the user's hand is captured when he/she 'pops' the first bubble, and the final position of the hand is captured when the user 'pops' the second bubble. The user's initial position, target position, trajectory information, and arm link lengths are calculated using the method discussed in [25] to: 1) determine the user's actual ROM, and 2) feed into the algorithm for determining the nominal baseline ROM value. For extraction of user data and calculating the user's actual ROM, the method discussed in [25] has been shown to output angle measurements that don't exceed $10 \%$ error as compared to ground truth measurements.

For this study, participants interacted with the system in their homes. The environment settings were maintained as a constant in order to maintain consistency. The virtual reality game screen was projected onto a large screen via a projector connected to a PC laptop. The chair height upon which the participant sat was $41 \mathrm{~cm}$ tall, the distance between the user's chair and the Kinect camera was $190 \mathrm{~cm}$, and the distance between the projector and the screen was $170 \mathrm{~cm}$. For each participant, a total of six reaching movements was collected per arm during game play.

\section{B. Results}

The purpose of the testing sessions was to collect elbow and shoulder range of motion (ROM) nominal values, and to compute a percent error by comparing the outcome metrics to the baseline generated by the described kinematic model. Typical evidence-based baseline models created by collecting human subjects' data as in [9] [10] [11], show an error ranging from $13.8 \%$ to $66.7 \%$ (for different kinematic measurements in [9]). For our methodology, we show an average error of $\mathbf{6 . 3 3}$ $\pm \mathbf{5 . 9 9 \%}$ for the elbow joint, $\mathbf{6 . 3 8} \pm \mathbf{4 . 7 4 \%}$ for the shoulder joint projected onto the coronal plane, and $\mathbf{1 1 . 3 6} \pm \mathbf{1 1 . 8 3} \%$ for the shoulder joint projected onto the sagittal plane; which falls in-line with human-data collection approaches.

Table 1 summarizes the comparison between the users' ROM nominal values versus the generated baseline values for a single trial. Taking into consideration the learning curve of the used platform, we show the resulting outcome metrics of the last trial of the participant's dominant arm. In this study, all participants are right hand dominant. Table 1 also shows the average errors of the three measurements made (including their respective standard deviations). It is important to mention that, although the presented task was the same for all participants, the state is correlated for each participant as defined by the corresponding body measurements. 
TABLE I. COMPARISON BETWEEN THE USERS' ROM VERSUS THE BASELINE VALUES FOR A SINGLE TRIAL.

\begin{tabular}{c|cc|cc|cc}
$\begin{array}{c}\text { Partici- } \\
\text { pants }\end{array}$ & \multicolumn{2}{|c|}{ Elbow ROM } & \multicolumn{2}{c|}{ Shoulder ROM (coronal) } & \multicolumn{2}{c}{ Shoulder ROM (sagittal) } \\
User [deg] & Error [\%] & User [deg] & Error [\%] & User [deg] & Error [\%] \\
\hline 1 & 27.45 & 9.84 & 76.15 & 9.90 & 47.57 & 4.65 \\
2 & 27.65 & 6.73 & 57.32 & 10.08 & 39.09 & 6.45 \\
3 & 7.38 & 15.83 & 33.42 & 4.76 & 45.25 & 35.59 \\
4 & 6.62 & 0.40 & 33.51 & 3.18 & 22.44 & 0.62 \\
5 & 27.38 & 7.13 & 23.44 & 14.13 & 24.56 & 22.77 \\
6 & 0.23 & 2.94 & 28.50 & 2.68 & 21.44 & 3.41 \\
7 & 16.93 & 19.09 & 40.08 & 6.28 & 71.22 & 27.35 \\
8 & 1.16 & 0.51 & 15.55 & 0.73 & 7.51 & 0.84 \\
9 & 2.92 & 3.59 & 53.34 & 14.33 & 20.32 & 0.90 \\
10 & 3.27 & 1.86 & 34.90 & 3.08 & 18.99 & 4.12 \\
11 & 5.06 & 1.75 & 48.24 & 1.03 & 56.42 & 18.25 \\
\hline Avg [\%] & & $\mathbf{6 . 3 3}$ & & $\mathbf{6 . 3 8}$ & & $\mathbf{1 1 . 3 6}$ \\
Std [\%] & & $\mathbf{5 . 9 9}$ & & $\mathbf{4 . 7 4}$ & & $\mathbf{1 1 . 8 3}$
\end{tabular}

TABLE II. PROGRESSION OF ROM ERRORS FOR THE ELBOW AND SHOULDER OF PARTICIPANT 1.

\begin{tabular}{c|ccc} 
& \multicolumn{3}{|c}{ RoM Errors [\%] } \\
Trials & Elbow & $\begin{array}{c}\text { Shoulder } \\
\text { (coronal) }\end{array}$ & $\begin{array}{c}\text { Shoulder } \\
\text { (sagittal) }\end{array}$ \\
\hline 1 & 9.44 & 9.96 & 8.76 \\
2 & 4.24 & 6.16 & 4.21 \\
3 & 3.66 & 8.72 & 14.02 \\
4 & 0.58 & 1.96 & 3.43 \\
5 & 11.40 & 7.61 & 8.34 \\
6 & 9.84 & 9.90 & 4.65 \\
\hline Avg [\%] & $\mathbf{6 . 5 3}$ & $\mathbf{7 . 3 9}$ & $\mathbf{7 . 2 3}$ \\
Std [\%] & $\mathbf{3 . 9 2}$ & $\mathbf{2 . 7 6}$ & $\mathbf{3 . 6 5}$
\end{tabular}

As stated earlier, the primary reason for using a baseline is to provide therapists a tool for objectively assessing improvements in a patient's motor skill in order to determine the effectiveness of a protocol. Table 2 shows the progression of the ROM errors for the elbow and shoulder of participant 1 over the six trials of their dominant hand. Figure 7 shows three boxplots to show the error variation of participant 1 for the joints of interest. As depicted, the average ROM errors for the three joints of interest are relatively low. The information shows the maximum, minimum, and median values for the ROM errors. This information is useful when assessing the kinematic performance of an individual and, if necessary, adapting the rehabilitation protocol to their needs. This highlights the additional benefit in using the modeling methodology to track a user's progress during sequential intervention trials.

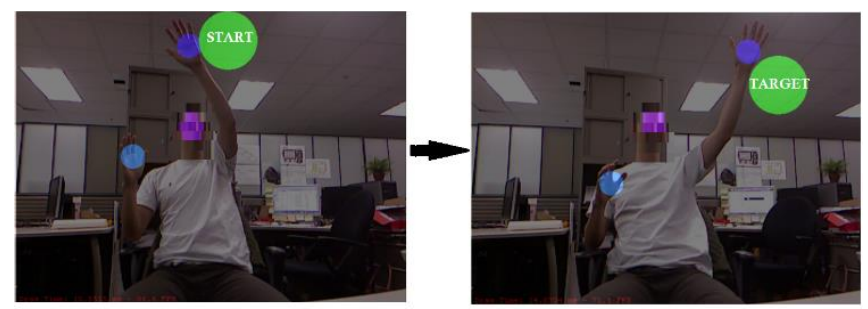

Figure 6. Example of a shoulder adduction movement from START to TARGET for a given task.

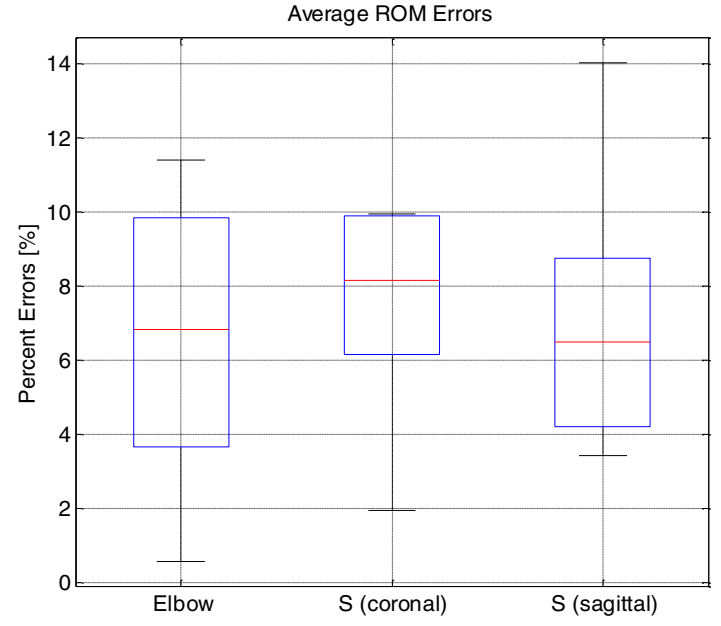

Figure 7. Boxplot showing the average ROM errors over six trials for participant 1 for each of the three joints of interest (elbow, shoulder in the coronal plane, and shoulder in the sagittal plane). 


\section{DISCUSSION AND FUTURE WORK}

From Table 1 we can see that participants moved relatively well as defined by the baseline generated by the proposed kinematic model. It seems that, on average, participants had the most trouble with the shoulder joint in the sagittal plane (average error of $\mathbf{1 1 . 3 6} \pm \mathbf{1 1 . 8 3 \%}$ ). From Table 2 and Figure 7, we can see that participant 1 had an average error of $\mathbf{6 . 5 3} \pm$ $\mathbf{3 . 9 2} \%$ for the elbow joint, $\mathbf{7 . 3 9} \pm \mathbf{2 . 7 6} \%$ for the shoulder joint in the coronal plane, and $\mathbf{7 . 2 3} \pm \mathbf{3 . 6 5} \%$ for the shoulder joint in the sagittal plane. Information such as this is important to the clinician, and in future systems such information could be transmitted to the therapist in charge in order to evaluate the population as a group or evaluate the individual independently.

One of the benefits of generating a baseline in real-time is the ability to modify it such that it calculates ground truth values for any kinematic parameter of interest. As previously mentioned, the proposed kinematic model generates an ideal trajectory as a function of the state of the current task. Most, if not all, kinematic parameters of interest can be computed from the final ideal trajectory. Namely: path length, deviation form path, accuracy, etc. This, coupled with the fact that the proposed kinematic model is able to compute a baseline depending on the user's capabilities, allows for a potential methodology that can be used to expand on existing rehabilitation systems for the in-home setting - one that can quantifiably and objectively assess upper-body movements.

\section{ACKNOWLEDGEMENT}

This work was supported in part by the NSF Graduate Research Fellowship under Grant No. DGE-1148903, and NSF Grant 1208287. Any opinions, findings, and conclusions or recommendations expressed in this material are those of the author's and do not necessarily reflect the views of the National Science Foundation.

\section{REFERENCES}

[1] M. Shaughnessy, B. M. Resnick, and R. F. Macko, "Testing a Model of Post-Stroke Exercise Behavior," Rehabilitation Nursing, Vol. 31, No. 1, pp. 15-21, 2006.

[2] H. I. Krebs, N. Hogan, M. L. Aisen, and B. T. Volpe, "Robot-aided Neurorehabilitation," Rehabilitation Engineering, IEEE Transactions on, Vol. 6, No. 1, pp. 75-87, 1998.

[3] B. T. Volpe, H. I. Krebs, N. Hogan, L. Edelstein, C. Diels, and M. Aisen, "A novel approach to stroke rehabilitation: Robot-aided sensorimotor stimulation," Neurology, Vol. 54, No. 10, pp. 1938-44, 2000.

[4] C. G. Burgar, P. S. Lum, P. C. Shor, and H. F. Van der Loos, "Development of robots for rehabilitation therapy: the Palo Alto VA/Stanford experience," Journal of Rehabilitation Research \& Development, Vol. 37, No. 6, 2000.

[5] J. C. C. Galloway, J. C. Ryu, and S. K. Agrawal, "Babies driving robots: self-generated mobility in very young infants," Intelligent Service Robotics, Vol. 1, No. 2, pp. 123-34, 2008.

[6] R. Colombo, F. Pisano, S. Micera, A. Mazzone, C. Delconte, M. C. Carrozza, P. Dario, and G. Minuco, "Upper limb rehabilitation and evaluation of stroke patients using robot-aided techniques," in Rehabilitation Robotics (ICORR), IEEE $9^{\text {th }}$ International Conference on, pp. 515-8, 2005.

[7] A. M. Howard, D. Brooks, E. Brown, A. Gebregiorgis, and Y. P. Chen, "Non-contact versus contact-based sensing methodologies for in-home upper arm robotic rehabilitation," in Rehabilitation Robotics (ICORR), IEEE International Conference on, pp. 1-6, 2013.

[8] D. Brooks, and A. M. Howard, "A Computational Method for Physical Rehabilitation Assessment," IEEE International Conference on Biomedical Robotics and Biomechatronics (BioRob), pp. 442-7, 2010.

[9] E. E. Butler, A. L. Ladd, S. A. Louie, L. E. LaMont, W. Wong, and J. Rose, "Three-dimensional kinematics of the upper limb during a Reach and Grasp Cycle for children," Gait \& Posture, Vol. 32, No. 1, pp. 72-7, 2010.

[10] M. Troke, A. P. Moore, F. J. Maillardet, and E. Cheek, "A normative database of lumber spine ranges of motion," Manual Therapy, Vol. 10, No. 3, pp. 198-206, 2005.

[11] D. H. Sutherland, R. A. Olshen, E. N. Biden, and M. P. Wyatt, "The development of mature walking," London: MacKeith Press, 1988.

[12] C. V. Granger, B. B. Hamilton, and F. S. Sherwin, "Guide for the user of the uniform data set for medical rehabilitation," Uniform data system for medical rehabilitation project, Buffalo General Hospital, New York, 1986.

[13] P. H. McCrea, J. J. Eng, and A. J. Hodgson, "Biomechanics of reaching: clinical implications for individuals with acquired brain injury," Disability \& Rehabilitation, Vol. 24, No. 10, pp. 534-41, 2002.

[14] R.M. Murray, and S.S. Sastry, "A Mathematical Introduction to Robotic Manipulators," CRC Press, 1994.

[15] M. Shimizu, H. Kakuya, W. K. Yoon, K. Kitagaki, and K. Kosuge, "Analytical inverse kinematic computation for 7-DOF redundant manipulators with joint limits and its application to redundancy resolution," Robotics, IEEE Transactions on, Vol. 24, No. 5, pp. 1131-42, 2008.

[16] B. Rohrer, S. Fasoli, H. I. Krebs, R. Hughes, B. Volpe, W. R. Frontera, J. Stein, and N. Hogan, "Movement smoothness changes during stroke recovery," The Journal of Neuroscience, Vol. 22, No. 18, pp. 9287-304, 2002.

[17] J. J. Chang, Y. S. Yang, W. L. Wu, L. Y. Guo, and F. C. Su, "The constructs of kinematic measures for reaching performance in stroke patients," Journal of Medicine and Biological Engineering, Vol. 28. No. 2, pp. 65-70, 2008.

[18] M. E. Morris, "Movement disorders in people with Parkinson disease: A model for physical therapy," Physical Therapy, Vol. 80, No. 6, pp. 578-87, 2000.

[19] Range of Motion (ROM)." Cambridge Dictionary of Human Biology and Evolution. N.p.: Cambridge UP, 2005. Credo Reference. 29 Aug. 2007. Web. 23 Feb. 2014

$<\mathrm{http} / / /$ search.credoreference.com/content/entry/cuphbe/range_of_mot ion_rom $/ 0>$.

[20] R.L. Gajdosik, and R.W. Bohannon, "Clinical Measurement of Range of Motion Review of Goniometry Emphasizing Reliability and Validity," Physical Therapy, Vol. 67, No. 12, pp. 1867-1872, 1987.

[21] R. F. Escamilla, S. W. Barrentine, G. S. Fleisig, N. Zheng, Y. Takada, D. Kingsley, and J. R. Andrews, "Pitching biomechanics as a pitcher approaches muscular fatigue during a simulated baseball game," The American Journal of Sports Medicine, Vol. 35, No. 1, pp. 23-33, 2007.

[22] I. L. Rojas, M. T. Provencher, S. Bhatia, K. C. Foucher, B. R. Bach, A. A. Romeo, M. A. Wimmer, and N. N. Verma, "Biceps activity during windmill softball pitching injury implications and comparison with overhand throwing," The American Journal of Sports Medice, Vol. 37, No. 3, pp. 558-65, 2009.

[23] S. García-Vergara, Y. P. Chen, and A. M. Howard, "Super Pop VR ${ }^{\mathrm{TM}}$. An adaptable virtual reality game for upper-body rehabilitation," in Virtual, Augmented, and Mixed Reality. Systems and Applications (Springer Berlin Heidelberg), pp. 40-9, 2013.

[24] S. García-Vergara, L. Brown, H. W. Park, and A. M. Howard, "Engaging Children in Play Therapy: The Coupling of Virtual Reality Games with Social Robotics," in Technologies of Inclusive Well-Being (Springer Berlin Heidelberg), pp. 139-63, 2014.

[25] M. E. Nixon, Y. P. Chen, and A. M. Howard, "Quantitative evaluation of the Microsoft Kinect for use in an upper extremity virtual rehabilitation environment," in Virtual Rehabilitation (ICVR), International Conference on, pp. 222-8, 2013. 